\title{
Controllability for transmission wave/plate equations on Riemannian manifolds
}

\author{
Li Deng* and Zhifei Zhang ${ }^{\dagger}$
}

\begin{abstract}
In this paper we consider controllability for transmission system of coupling wave equations with Euler-Bernoulli equations on Riemannian manifolds. We show that such a system is exactly controllable by boundary controls only along the exterior boundary, which means there is no control on the interface of the transmission system. Our proofs rely on the Hilbert Uniqueness Method(HUM) and geometric multiplier method.
\end{abstract}

Keywords: exact controllability; transmission problem; Riemannian geometry method.

\section{Introduction}

Transmission systems appear in many practical control systems such as chemical reactions coupling, structural-acoustic systems and many other interactive physical processes. Among these systems are the transmission problem of hyperbolic systems, which attract much attention and have strong physical backgrounds, for example, it can describe the displacement of flexible structures consisting of two physically different types of materials. The analysis of controllability and stabilization for the transmission of hyperbolic systems has been widely carried out.

In this article we aim to discuss the controllability of transmission system of coupling wave equations with Euler-Bernoulli equations on Riemannian manifolds, of which system we considered the stabilization property in our recent work [24]. More precisely, we consider the case where we only introduce the control on the exterior boundary. That means there is no control on the interface of the transmission problem. We prove that the system is exactly controllable under some geometric assumptions on the interface. We found that such kind of geometric assumption can not be removed. The reason is that, the system may not be controllable only from exterior boundaries, due to total reflection at the interface, as already pointed out by many other authors, see [15, 17].

${ }^{*}$ School of Mathematics, Southwest Jiaotong University, Chengdu, 611756, China. This author was supported by the National Science Foundation of China, grant no. 11401491.

${ }^{\dagger}$ Department of Mathematics, Huazhong University of Science and Technology, Wuhan, 430074, China. Email: zhangzf@hust.edu.cn. This author was supported by the National Science Foundation of China under Grant 61473126 . 


\subsection{Statement of the problem and the main result}

Let $M$ be a complete two dimensional Riemannian manifold of class $C^{3}$ with $C^{3}$-metric $g(\cdot, \cdot)=\langle\cdot, \cdot\rangle$. For each $x \in M, M_{x}$ is the tangential space of $M$ at $x$. Denote the set of all $n$ order tensor fields on $M$ by $T^{n}(M)=\cup_{x \in M} T_{x}^{n}(M)$, where $n$ is a nonnegative integer. It is well known that the space $T_{x}^{n}(M)$ of $n$ order tensor on $M_{x}$ is an inner product space. Its inner product is defined by

$$
\left\langle T_{1}, T_{2}\right\rangle_{T_{x}^{n}}=\sum_{i_{1}, i_{2}, \cdots, i_{n}=1}^{2} T_{1}\left(e_{i_{1}}, \cdots, e_{i_{n}}\right) T_{2}\left(e_{i_{1}}, \cdots, e_{i_{n}}\right) \text { at } x,
$$

for any $T_{1}, T_{2} \in T_{x}^{n}(M)$, where $e_{1}, e_{2}$ is an orthonormal basis of $M_{x}$ for $x \in M$. For any $T \in T^{2}(M)$, the trace of $T$ is defined by

$$
\operatorname{tr} T=\sum_{i=1}^{2} T\left(e_{i}, e_{i}\right)
$$

We denote by $\nabla$ the gradient, by $D$ the Levi-Civita connection and by $\Delta=\operatorname{div}(\nabla)$ the Laplace-Beltrami operator in the Riemannian metric $g$. For any vector field $H$ on $M, D H$ is the covariant differential of $H$ which is a second order tensor field in the following sense:

$$
D H(X, Y)=D_{Y} H(X)=\left\langle D_{Y} H, X\right\rangle \text { for all } X, Y \in M_{x}, x \in M .
$$

For scalar function $u$ we have $D u=\nabla u$.

Let $\Omega$ be an open, bounded, connected subset of $M$ with smooth boundary $\Gamma$ such that $\bar{\Omega}=\bar{\Omega}_{1} \cup \bar{\Omega}_{2}$, where $\Omega_{i}, i=1,2$ are two disjoint open connected bounded domains with smooth boundary. They satisfy that $\bar{\Omega}_{1} \cap \bar{\Omega}_{2}=S$, $\partial \Omega_{1}=S \cup \Gamma_{1}$ and $\partial \Omega_{2}=S \cup \Gamma_{2}$ (See Figure 1).

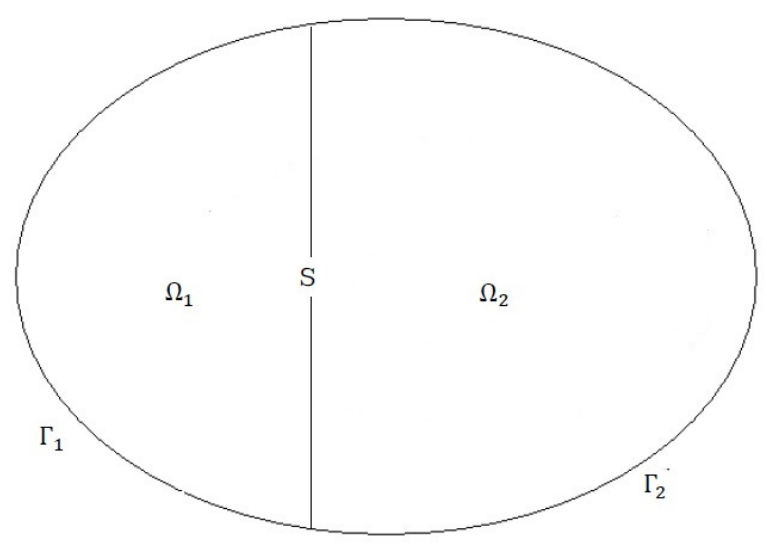

Figure 1: the domain 
We consider the wave equation in $\Omega_{1}$ coupled with the Euler-Bernoulli plate equation in $\Omega_{2}$ by the interface $S$ with boundary controls $\phi(x, t), \psi(x, t), \zeta(x, t)$ on $\Gamma_{0} \subset \Gamma$ which is a subset of the exterior boundary $\Gamma$. More precisely, we consider the following system:

$$
\begin{cases}\partial_{t}^{2} u_{1}(x, t)-\Delta u_{1}(x, t)=0, & \text { in } \Omega_{1} \times(0,+\infty), \\ \partial_{t}^{2} u_{2}(x, t)+\Delta^{2} u_{2}(x, t)-(1-\mu) \operatorname{div}\left(\mathcal{K} \nabla u_{2}\right)(x, t)=0, & \text { in } \Omega_{2} \times(0,+\infty), \\ u_{i}(x, 0)=u_{i}^{0}(x), \partial_{t} u_{i}(x, 0)=u_{i}^{1}(x), & \text { in } \Omega_{i}, i=1,2, \\ u_{1}=u_{2}, B_{1} u_{2}=0, B_{2} u_{2}=\partial_{\nu_{1}} u_{1}, & \text { on } S \times(0,+\infty), \\ u_{1}=0, & \text { on }\left(\Gamma_{1} / \Gamma_{0}\right) \times(0,+\infty), \\ u_{1}=\phi, & \text { on }\left(\Gamma_{1} \cap \Gamma_{0}\right) \times(0,+\infty), \\ u_{2}=\partial_{\nu_{2}} u_{2}=0, & \text { on }\left(\Gamma_{2} / \Gamma_{0}\right) \times(0,+\infty), \\ u_{2}=\psi, & \text { on }\left(\Gamma_{2} \cap \Gamma_{0}\right) \times(0,+\infty), \\ \partial_{\nu_{2}} u_{2}=\zeta, & \text { on }\left(\Gamma_{2} \cap \Gamma_{0}\right) \times(0,+\infty),\end{cases}
$$

where $\mathcal{K}$ is the Gaussian curvature function on $\Omega_{2}$. Here $\nu_{i}=\nu_{i}(x)$ denotes the unit outward normal vector of $\Omega_{i}$ along $\partial \Omega_{i}=\Gamma_{i} \cup S$ for $i=1,2$. In the above system, $0<\mu<\frac{1}{2}$ is the Poisson coefficient, and the boundary operators $B_{1}, B_{2}$ are defined on $\partial \Omega_{2}=\Gamma_{2} \cup S$ as follows:

$$
\begin{aligned}
& B_{1} y=\Delta y-(1-\mu) D^{2} y\left(\tau_{2}, \tau_{2}\right) \\
& B_{2} y=\partial_{\nu_{2}} \Delta y+(1-\mu) \partial_{\tau_{2}}\left(D^{2} y\left(\tau_{2}, \nu_{2}\right)\right)+\mathcal{K} \partial_{\nu_{2}} y
\end{aligned}
$$

where $D^{2} y$ is the Hessian of $y$ and $\tau_{2}$ is the unit tangential vector along the boundary $\partial \Omega_{2}=\Gamma_{2} \cup S$.

Remark 1.1. The term $(1-\mu) \operatorname{div}\left(\mathcal{K} \nabla u_{2}\right)$ in the system (1.4) comes from the curvedness of the Riemannian metric $g$. For details, see [22, Model, pp.150]

To obtain the controllability of the problem (1.4), the following geometrical hypotheses are assumed:

Geometrical assumptions Given the triple $\left\{\Omega_{1}, S, \Omega_{2}\right\}$, there exists a vector field $H$ on Riemannian manifold $(M, g)$ such that the following three properties hold true:

(A.1) $D H(\cdot, \cdot)$ is strictly positive definite on $\bar{\Omega}$ : there exists a constant $\rho>0$ such that for all $x \in \bar{\Omega}$, for all $X \in M_{x}$ (the tangent space at $x$ ):

$$
D H(X, X) \equiv\left\langle D_{X} H, X\right\rangle \geq \rho|X|^{2} .
$$

(A.2) The control area $\Gamma_{0} \triangleq \Gamma_{0}^{1} \cup \Gamma_{0}^{2}$ satisfies

$$
\Gamma_{0}^{i}=\left\{x \in \Gamma_{i} \quad \mid\left\langle H, \nu_{i}\right\rangle>0 \text { on } \Gamma_{i}, i=1,2\right\} .
$$

(A.3) The interface satisfies

$$
\left\langle H, \nu_{i}\right\rangle=0 \text {, on } S \text {. }
$$


Remark 1.2. For any Riemannian manifold $M$, the existence of such a vector field $H$ in (A.1) has been proved in [21], where some examples are given, too. See also [22]. In the framework of Euclidean metric, one can take the vector field $H=x-x_{0}$. This was given first in the paper [11] as well as in $[12,13]$. Thus $D H(X, X)=|X|^{2}$ follows, which means assumption (A.1) always holds true with $\rho=1$ for the Euclidean case.

Here we state our main result.

Theorem 1.1. We assume the geometrical assumptions (A.1), (A.2) and (A.3) hold true. Then the transmission problem (1.4) is exactly $L^{2}\left(\Omega_{1}\right) \times L^{2}\left(\Omega_{2}\right) \times H^{-1}\left(\Omega_{1}\right) \times H^{-2}\left(\Omega_{2}\right)$ controllable by $L^{2}\left(0, T ; L^{2}\left(\Gamma_{0}^{1}\right)\right) \times L^{2}\left(0, T ; L^{2}\left(\Gamma_{0}^{2}\right)\right) \times L^{2}\left(0, T ; L^{2}\left(\Gamma_{0}^{2}\right)\right)$ controls, i.e., there exist some $T>T_{0}$, control functions $\phi \in L^{2}\left(0, T ; L^{2}\left(\Gamma_{0}^{1}\right)\right), \psi \in L^{2}\left(0, T ; L^{2}\left(\Gamma_{0}^{2}\right)\right)$ and $\zeta \in L^{2}\left(0, T ; L^{2}\left(\Gamma_{0}^{2}\right)\right)$ such that the corresponding solution $\left(u_{1}, u_{2}, \partial_{t} u_{1}, \partial_{t} u_{2}\right)$ of (1.4) satisfies

$$
\left(u_{1}, u_{2}\right)(\cdot, T)=0,\left(\partial_{t} u_{1}, \partial_{t} u_{2}\right)(\cdot, T)=0
$$

where $\Gamma_{0}^{i}$ are given in (1.6) and

$$
T_{0}=\frac{8}{\rho} \sup _{x \in \Omega_{1}}|H|
$$

\section{$1.2 \quad$ Literature}

Controllability for transmission problems were studied by several authors, for example, for the coupled wave equations with constant coefficients, the controllability results are presented in [15] by applying the Hilbert Uniqueness Method (HUM). For the coupled wave equations with variable coefficients (describing the wave with variable propagation speed), the boundary controllability is treated in [10], which involves the research for transmission problem of anisotropic elastic materials. The exact controllability for transmission plate equations was addressed in [16]. We refer to $[3,7,9,18,19]$ for related results on transmission problems of other hyperbolic systems.

These works mentioned above offer fruitful results regarding the transmission systems in the framework of Euclidean metric. However, the problems on the compact Riemannian manifolds have limited results compared with the problems on Euclidean spaces. The main difficulty comes from the fact that the trapped geodesics in the general Riemannian manifolds can preclude the effectiveness of the canonical multiplier which plays an important role in obtaining the global estimates in the controllability analysis. At the same time, the transmission problems on Riemannian manifolds have strong practical applications. For example, once the plates in $[1,2]$ have curved middle surfaces $\Omega$, the transmission system considered by $[1,2]$ becomes the one on the general Riemannian manifold $(\Omega, g)$, where $g$ is the induced Riemannian metric.

In the present paper we consider the controllability of a coupled wave-plate system on Riemannian manifolds. The main tool we use is the geometric multiplier method, which first appeared in [21] and subsequently in [4, 6, 14, 23, 24], and many others. First, we establish multiplier equality for the coupled wave-plate system on Riemannian manifolds. Then, under the geometric assumptions on the domain, we obtain the controllability of the coupled system in Theorem 1.1. 
The content of this paper is organized as follows. In Section 2, we establish the multiplier equality for the dual transmission system by the geometric multiplier method. Finally in Section 3, we present the proofs of the main results.

\section{The dual transmission system.}

In this section, we will discuss the dual transmission system.

$$
\begin{cases}\partial_{t}^{2} w_{1}(x, t)-\Delta w_{1}(x, t)=0, & \text { in } \Omega_{1} \times(0,+\infty), \\ \partial_{t}^{2} w_{2}(x, t)+\Delta^{2} w_{2}(x, t)-(1-\mu) \operatorname{div}\left(\mathcal{K} \nabla w_{2}\right)(x, t)=0, & \text { in } \Omega_{2} \times(0,+\infty), \\ w_{i}(x, 0)=w_{i}^{0}(x), \partial_{t} w_{i}(x, 0)=w_{i}^{1}(x), & \text { in } \Omega_{i}, i=1,2, \\ w_{1}=w_{2}, B_{1} w_{2}=0, B_{2} w_{2}=\partial_{\nu_{1}} w_{1}, & \text { on } S \times(0,+\infty), \\ w_{1}=0, & \text { on } \Gamma_{1} \times(0,+\infty), \\ w_{2}=\partial_{\nu_{2}} w_{2}=0, & \text { on } \Gamma_{2} \times(0,+\infty) .\end{cases}
$$

First, we write the system (2.1) as an abstract Cauchy problem to treat its well-posedness. Let

$$
\mathcal{H}=\left\{\left(w_{1}, v_{1}, w_{2}, v_{2}\right) \in H^{1}\left(\Omega_{1}\right) \times L^{2}\left(\Omega_{1}\right) \times H^{2}\left(\Omega_{2}\right) \times L^{2}\left(\Omega_{2}\right),\left.w_{1}\right|_{S}=\left.w_{2}\right|_{S}\right\}
$$

equipped with the inner product

$$
\begin{aligned}
& \left\langle\left(w_{1}, v_{1}, w_{2}, v_{2}\right),\left(y_{1}, z_{1}, y_{2}, z_{2}\right)\right\rangle_{\mathcal{H}} \\
= & \int_{\Omega_{1}}\left(\left\langle D w_{1}, D y_{1}\right\rangle+v_{1} z_{1}\right) d x+\int_{\Omega_{2}}\left(a\left(w_{2}, y_{2}\right)+v_{2} z_{2}\right) d x,
\end{aligned}
$$

where the bilinear form $a(\cdot, \cdot)$ is defined on $H^{2}\left(\Omega_{2}\right) \times H^{2}\left(\Omega_{2}\right)$ by

$$
a(y, z)=(1-\mu)\left\langle D^{2} y, D^{2} z\right\rangle_{T_{x}^{2}}+\mu \Delta y \Delta z .
$$

Here the inner product $\langle\cdot, \cdot\rangle_{T_{x}^{2}}$ is given in (1.1).

Then we define the operator $\mathcal{A}$ in $\mathcal{H}$ by

$$
\left(\begin{array}{cccc}
0 & I d & 0 & 0 \\
\Delta & 0 & 0 & 0 \\
0 & 0 & 0 & I d \\
0 & 0 & -\Delta^{2}+(1-\mu) \operatorname{div}(\mathcal{K} \nabla) & 0
\end{array}\right)
$$

with the domain

$$
\mathcal{D}(\mathcal{A})=\left\{\begin{array}{c|c}
\left(v_{1}, \Delta w_{1}, v_{2},-\Delta^{2} w_{2}\right) \in \mathcal{H} \\
\left(w_{1}, v_{1}, w_{2}, v_{2}\right) \in \mathcal{H} \mid \begin{array}{c}
B_{1} w_{2}=0, B_{2} w_{2}=\partial_{\nu_{1}} w_{1}, \text { on } S, \\
w_{1}=0, \text { on } \Gamma_{1}, \\
w_{2}=\partial_{\nu_{2}} w_{2}=0, \text { on } \Gamma_{2} .
\end{array}
\end{array}\right\}
$$

By the semigroup theory, we can show that the operator $(\mathcal{A}, \mathcal{D}(\mathcal{A}))$ defined by $(2.4)$ and (2.5) generates a $C_{0}$-group on the Hilbert space $\mathcal{H}$ endowed with the inner product (2.2). Thus we can obtain the well-posedness for the problem (2.1)(See Proposition 2.1). Here we state this proposition without proof. Its proof is similar as that in [1]. 
Proposition 2.1. For all given initial data $\left(w_{1}^{0}, w_{1}^{1}, w_{2}^{0}, w_{2}^{1}\right) \in \mathcal{H}$, the problem (2.1) admits a unique global weak solution

$$
\left(w_{1}, \partial_{t} w_{1}, w_{2}, \partial_{t} w_{2}\right) \in C([0, \infty) ; \mathcal{D}(\mathcal{A})) \cap C^{1}([0, \infty), \mathcal{H}) .
$$

We define the energy of a solution

$$
w=\left\{\begin{array}{l}
w_{1}, x \in \Omega_{1} \\
w_{2}, x \in \Omega_{2}
\end{array}\right.
$$

of the system (2.1) by

$$
E(t)=E_{1}(t)+E_{2}(t)
$$

where we denote

$$
E_{1}(t)=\frac{1}{2} \int_{\Omega_{1}}\left(\left(\partial_{t} w_{1}(x, t)\right)^{2}+\left|D w_{1}(x, t)\right|^{2}\right) d x,
$$

and

$$
E_{2}(t)=\int_{\Omega_{2}}\left(\left(\partial_{t} w_{2}(x, t)\right)^{2}+a\left(w_{2}, w_{2}\right)\right) d x .
$$

To get some technical estimates for the dual system (2.1), in what follows we assume that $w$ is a strong solution. By a classical density argument we can see that these estimates hold for a a weak solution as well.

The following formula presents the relationship between the interior terms and boundary terms.

Lemma 2.2. [8, Lemma 3.1, p.55] Let $y, v \in H^{4}\left(\Omega_{2}\right)$. Then we have

$$
\int_{\Omega_{2}}\left(\Delta^{2} y-(1-\mu) d i v(\mathcal{K} \nabla y)\right) v d x=\int_{\Omega_{2}} a(y, v) d x-\int_{\partial \Omega_{2}} B_{1} y \partial_{\nu_{2}} v d \sigma+\int_{\partial \Omega_{2}} B_{2} y v d \sigma .
$$

For the energy $E(t)$ defined in (2.7), we have

Lemma 2.3. For any $w$ solves the dual system (2.1), we have

$$
\partial_{t} E(t)=0 .
$$

Lemma 2.4. Let $H$ be a vector field on $\Omega_{2}$. For any $w_{2}$ solves the plate equation on $\Omega_{2}$ as

$$
\partial_{t}^{2} w_{2}(x, t)+\Delta^{2} w_{2}(x, t)-(1-\mu) \operatorname{div}\left(\mathcal{K} \nabla w_{2}\right)(x, t)=0,
$$

we have

$$
\begin{aligned}
\int_{0}^{T} \int_{\Gamma_{2} \cup S} \mathfrak{B}_{2}\left(w_{2}\right) d x d t= & \left.2 \int_{\Omega_{2}} \partial_{t} w_{2} H\left(w_{2}\right) d x\right|_{0} ^{T}+\int_{0}^{T} \int_{\Omega_{2}}\left[\left(\partial_{t} w_{2}\right)^{2}-a\left(w_{2}, w_{2}\right)\right] d i v H d \sigma d t \\
& +4(1-\mu) \int_{0}^{T} \int_{\Omega_{2}} D H\left(D^{2} w_{2}, D^{2} w_{2}\right) d x d t+4 \mu \int_{0}^{T} \int_{\Omega_{2}} D H\left(\Delta w_{2}, \Delta w_{2}\right) \\
& +2(1-\mu) \int_{0}^{T} \int_{\Omega_{2}}\left\langle D^{2} w_{2}, l\left(w_{2}\right)\right\rangle d x d t \\
& +2 \mu \int_{0}^{T} \int_{\Omega_{2}} \Delta w_{2} \cdot \operatorname{trl}\left(w_{2}\right) d x d t
\end{aligned}
$$


where the boundary term

$$
\mathfrak{B}_{2}\left(w_{2}\right)=2 B_{1}\left(w_{2}\right) \partial_{\nu_{2}}\left(H\left(w_{2}\right)\right)-2 H\left(w_{2}\right) B_{2}\left(w_{2}\right)+\left[\left(\partial_{t} w_{2}\right)^{2}-a\left(w_{2}, w_{2}\right)\right]\left\langle H, \nu_{2}\right\rangle,
$$

and

$$
l\left(w_{2}\right)=-\mathbf{R}\left(D w_{2}, \cdot, H, \cdot\right)-D^{2} H\left(D w_{2}, \cdot, \cdot\right) .
$$

Here $\mathbf{R}$ is the curvature tensor of the Levi-Civita connection D (for details see [20]).

Proof. Multiplying the plate equation $(2.10)$ by $2 H\left(w_{2}\right)$ yields

$$
2 \partial_{t}^{2} w_{2}(x, t) H\left(w_{2}\right)+2\left(\Delta^{2} w_{2}(x, t)-(1-\mu) \operatorname{div}\left(\mathcal{K} \nabla w_{2}\right)(x, t)\right) H\left(w_{2}\right)=0 .
$$

Using the divergence formula we have

$$
2 \partial_{t}^{2} w_{2}(x, t) H\left(w_{2}\right)=2 \partial_{t}\left[\partial_{t} w_{2} H\left(w_{2}\right)\right]-\operatorname{div}\left[\left(\partial_{t} w_{2}\right)^{2} H\right]+\left(\partial_{t} w_{2}\right)^{2} \operatorname{div} H
$$

From Lemma 2.2 we know

$$
\begin{aligned}
& \int_{0}^{T} \int_{\Omega_{2}}\left(\Delta^{2} w_{2}-(1-\mu) \operatorname{div}\left(\mathcal{K} \nabla w_{2}\right)\right) 2 H\left(w_{2}\right) d x \\
= & 2 \int_{0}^{T} \int_{\Omega_{2}} a\left(w_{2}, H\left(w_{2}\right)\right) d x-2 \int_{0}^{T} \int_{\partial \Omega_{2}}\left(B_{1} w_{2} \partial_{\nu_{2}}\left(H\left(w_{2}\right)\right)-B_{2} w_{2} H\left(w_{2}\right)\right) d \sigma .
\end{aligned}
$$

Now we deal with the term $\int_{\Omega_{2}} a\left(w_{2}, H\left(w_{2}\right)\right) d x$. Since $D^{2} w_{2}$ is a symmetric second order tensor field, we have from [23, Lemma 2.7] that

$$
\left\langle D^{2} y, D^{2}(H(y))\right\rangle_{T_{x}^{2}}=\frac{1}{2} H\left(\left|D^{2} y\right|_{T_{x}^{2}}^{2}\right)+2 D H\left(D^{2} y, D^{2} y\right)+\left\langle D^{2} y, l(y)\right\rangle_{T_{x}^{2}}
$$

and

$$
\operatorname{tr} D^{2} y \cdot \operatorname{tr} D^{2}(H(y))=\frac{1}{2} H\left((\Delta y)^{2}\right)+2 D H(\Delta y, \Delta y)+\Delta w_{2} \cdot \operatorname{trl}(y),
$$

where $l(y)$ is a lower order term respect to $a\left(w_{2}, w_{2}\right)$ given in $(2.13)$ and " . denotes the position of the variable. Combining the equalities $(2.17),(2.18)$ and the definition of $a(y, z)$ in (2.3), we can easily have

$$
\begin{aligned}
a\left(w_{2}, H\left(w_{2}\right)\right)= & \frac{1}{2} H\left(a\left(w_{2}, w_{2}\right)\right)+2(1-\mu) D H\left(D^{2} w_{2}, D^{2} w_{2}\right)+2 \mu D H\left(\Delta w_{2}, \Delta w_{2}\right) \\
& +(1-\mu)\left\langle D^{2} w_{2}, l\left(w_{2}\right)\right\rangle_{T_{x}^{2}}+\mu \Delta w_{2} \operatorname{trl}\left(w_{2}\right) .
\end{aligned}
$$

Note that

$$
\int_{\Omega_{2}} H\left(a\left(w_{2}, w_{2}\right)\right) d x=\int_{\partial \Omega_{2}} a\left(w_{2}, w_{2}\right)\left\langle H, \nu_{2}\right\rangle d \sigma-\int_{\Omega_{2}} a\left(w_{2}, w_{2}\right) \operatorname{div} H d \sigma,
$$


thus we have

$$
\begin{aligned}
\int_{\Omega_{2}} a\left(w_{2}, H\left(w_{2}\right)\right) d x= & \frac{1}{2} \int_{\partial \Omega_{2}} a\left(w_{2}, w_{2}\right)\left\langle H, \nu_{2}\right\rangle d \sigma-\frac{1}{2} \int_{\Omega_{2}} a\left(w_{2}, w_{2}\right) \operatorname{div} H d x \\
& +2(1-\mu) \int_{\Omega_{2}} D H\left(D^{2} w_{2}, D^{2} w_{2}\right) d x+2 \mu \int_{\Omega_{2}} D H\left(\Delta w_{2}, \Delta w_{2}\right) d x \\
& +(1-\mu) \int_{\Omega_{2}}\left\langle D^{2} w_{2}, l\left(w_{2}\right)\right\rangle_{T_{x}^{2}} d x+\mu \int_{\Omega_{2}} \Delta w_{2} \operatorname{trl}\left(w_{2}\right) d x .
\end{aligned}
$$

Integrating the equality $(2.15)$ on $(0, T) \times \Omega_{2}$ and combining the equalities $(2.16),(2.20)$ to obtain the desired equality (2.11).

Lemma 2.5. Let $p$ be a function. For any $w_{2}$ solves the plate equation given in Lemma 2.4, we have

$$
\begin{aligned}
\int_{0}^{T} \int_{\Gamma_{2} \cup S} \mathfrak{B}_{2}^{\prime}\left(w_{2}\right) d x d t= & \left.\int_{\Omega_{2}} p \partial_{t} w_{2} w_{2} d x\right|_{0} ^{T}-\int_{0}^{T} \int_{\Omega_{2}}\left[\left(\partial_{t} w_{2}\right)^{2}-a\left(w_{2}, w_{2}\right)\right] p d x d t \\
& +\int_{0}^{T} \int_{\Omega_{2}}\left[2(1-\mu) D^{2} w_{2}\left(D p, D w_{2}\right)+2 \mu \Delta w_{2}\left\langle D p, D w_{2}\right\rangle\right] d x d t \\
& +\int_{0}^{T} \int_{\Omega_{2}} w_{2} a\left(p, w_{2}\right) d x d t
\end{aligned}
$$

where the boundary term

$$
\mathfrak{B}_{2}^{\prime}\left(w_{2}\right)=B_{1}\left(w_{2}\right) \partial_{\nu_{2}}\left(p w_{2}\right)-p w_{2} B_{2}\left(w_{2}\right)
$$

Proof. We use the multiplier $p w_{2}$. A similar computation leads to the above equality (2.21).

Lemma 2.6. Let $H$ be a vector field on $\Omega_{1}$. For any $w_{1}$ solves the wave equation on $\Omega_{1}$ as

$$
\partial_{t}^{2} w_{1}(x, t)-\Delta w_{1}(x, t)=0,
$$

we have

$$
\begin{aligned}
\int_{0}^{T} \int_{\Gamma_{1} \cup S} \mathfrak{B}_{1}\left(w_{1}\right) d x d t= & \left.2 \int_{\Omega_{1}} \partial_{t} w_{1} H\left(w_{1}\right) d x\right|_{0} ^{T}+\int_{0}^{T} \int_{\Omega_{1}}\left[\left(\partial_{t} w_{1}\right)^{2}-\left|D w_{1}\right|^{2}\right] d i v H d x d t \\
& +2 \int_{0}^{T} \int_{\Omega_{1}} D H\left(D w_{1}, D w_{1}\right) d x d t
\end{aligned}
$$

where the boundary term

$$
\mathfrak{B}_{1}\left(w_{1}\right)=2 \partial_{\nu_{1}} w_{1} H\left(w_{1}\right)+\left[\left(\partial_{t} w_{1}\right)^{2}-\left|D w_{1}\right|^{2}\right]\left\langle H, \nu_{1}\right\rangle .
$$


Proof. Using the identities in [21, Theorem 2.1] we have

$$
\begin{aligned}
2 \int_{\Omega_{1}} \Delta w_{1} H\left(w_{1}\right) d x & =2 \int_{\partial \Omega_{1}} H\left(w_{1}\right) \partial_{\nu_{1}} w_{1} d \sigma-2 \int_{\Omega_{1}} D H\left(D w_{1}, D w_{1}\right) d x \\
& -\int_{\partial \Omega_{1}}\left|D w_{1}\right|^{2}\left\langle H, \nu_{1}\right\rangle d \sigma+\int_{\Omega_{1}}\left|D w_{1}\right|^{2} \operatorname{div} H(x) d x
\end{aligned}
$$

Similar to the identity (2.15), we have

$$
\begin{aligned}
2 \int_{0}^{T} \int_{\Omega_{1}} \partial_{t}^{2} w_{1}(x, t) H\left(w_{1}\right) d x d t= & \left.2 \int_{\Omega_{1}}\left[\partial_{t} w_{1} H\left(w_{1}\right)\right] d x\right|_{0} ^{T}-\int_{0}^{T} \int_{\partial \Omega_{1}}\left(\partial_{t} w_{1}\right)^{2}\left\langle H, \nu_{1}\right\rangle d \sigma d t \\
& +\int_{0}^{T} \int_{\Omega_{1}}\left(\partial_{t} w_{1}\right)^{2} \operatorname{div} H d x d t
\end{aligned}
$$

Combining the identities (2.25) and (2.26) to complete the proof.

Lemma 2.7. Let $p$ be a function on $\Omega_{1}$. For any $w_{1}$ solves the wave equation given in Lemma 2.6, we have

$$
\begin{aligned}
\int_{0}^{T} \int_{\Gamma_{1} \cup S} \mathfrak{B}_{1}^{\prime}\left(w_{1}\right) d x d t= & \left.\int_{\Omega_{1}} \partial_{t} w_{1} p w_{1} d x\right|_{0} ^{T}-\int_{0}^{T} \int_{\Omega_{1}} p\left[\left(\partial_{t} w_{1}\right)^{2}-\left|D w_{1}\right|^{2}\right] d x d t \\
& +\int_{0}^{T} \int_{\Omega_{1}} w_{1}\left\langle D p, D w_{1}\right\rangle d x d t
\end{aligned}
$$

where the boundary term

$$
\mathfrak{B}_{1}^{\prime}\left(w_{1}\right)=\partial_{\nu_{1}} w_{1} p w_{1}
$$

Proof. We use the multiplier $p w_{1}$. A similar computation as that in Lemma 2.6 leads to the above equality (2.27).

\section{Proof of exact controllability result: Theorem 1.1.}

By Hilbert uniqueness method (HUM)(see e.g. [15]), we know that the exact controllability of system (1.4) is equivalent to the observability inequality of system (2.1). Therefore, we only need to establish the following observability result.

Theorem 3.1. We assume the geometrical assumptions (A.1), (A.2) and (A.3) hold true. Let $T_{0}=8 \rho^{-1} \sup _{x \in \Omega_{1}}|H|$. Then for all $T>T_{0}$ there exists a constant $c_{T}>0$ such that

$$
c_{T} E(0) \leq \int_{0}^{T} \int_{\Gamma_{2} \cap \Gamma_{0}}\left(D^{2} w_{2}\left(\nu_{2}, \nu_{2}\right)\right)^{2} d \sigma d t+\int_{0}^{T} \int_{\Gamma_{1} \cap \Gamma_{0}}\left(\partial_{\nu_{1}} w_{1}\right)^{2} d \sigma d t
$$

for all solutions w to problem (2.1). 
Step 1. Estimates of the plate part on $\Omega_{2}$.

Using Lemma 2.4 and Lemma 2.5, we obtain

$$
\begin{aligned}
& \int_{0}^{T} \int_{\Gamma_{2} \cup S}\left(\mathfrak{B}_{2}\left(w_{2}\right)+\mathfrak{B}_{2}^{\prime}\left(w_{2}\right)\right) d x d t \\
= & \left.\int_{\Omega_{2}} \partial_{t} w_{2}\left(2 H\left(w_{2}\right)+\left(\operatorname{div} H-\frac{3}{2} \rho\right) w_{2}\right) d x\right|_{0} ^{T}+\frac{3}{2} \rho \int_{0}^{T} \int_{\Omega_{2}}\left[\left(\partial_{t} w_{2}\right)^{2}-a\left(w_{2}, w_{2}\right)\right] d \sigma d t \\
& +4(1-\mu) \int_{0}^{T} \int_{\Omega_{2}} D H\left(D^{2} w_{2}, D^{2} w_{2}\right) d x d t+4 \mu \int_{0}^{T} \int_{\Omega_{2}} D H\left(\Delta w_{2}, \Delta w_{2}\right) d x d t \\
& +2(1-\mu) \int_{0}^{T} \int_{\Omega_{2}}\left\langle D^{2} w_{2}, l\left(w_{2}\right)\right\rangle d x d t+2 \mu \int_{0}^{T} \int_{\Omega_{2}} \Delta w_{2} \cdot \operatorname{trl}\left(w_{2}\right) d x d t \\
& +\int_{0}^{T} \int_{\Omega_{2}}\left[2(1-\mu) D^{2} w_{2}\left(D(\operatorname{div} H), D w_{2}\right)+2 \mu \Delta w_{2}\left\langle D(\operatorname{div} H), D w_{2}\right\rangle\right] d x d t \\
& +\int_{0}^{T} \int_{\Omega_{2}} w_{2} a\left(\operatorname{div} H, w_{2}\right) d x d t
\end{aligned}
$$

where we set $p=\operatorname{div} H-\frac{3}{2} \rho$ in Lemma 2.5. Then by Cauchy-Schwartz inequality we have

$$
\begin{aligned}
& \left.\int_{\Omega_{2}} \partial_{t} w_{2}\left(2 H\left(w_{2}\right)+\left(\operatorname{div} H-\frac{3}{2} \rho\right) w_{2}\right) d x\right|_{0} ^{T} \\
\geq & -\varepsilon\left(E_{2}(T)+E_{2}(0)\right)-C_{\varepsilon}\left(\left\|D w_{2}(0)\right\|^{2}+\left\|D w_{2}(T)\right\|^{2}+\left\|w_{2}(0)\right\|^{2}+\left\|w_{2}(T)\right\|^{2}\right), \\
& 2(1-\mu) \int_{0}^{T} \int_{\Omega_{2}}\left\langle D^{2} w_{2}, l\left(w_{2}\right)\right\rangle d x d t+2 \mu \int_{0}^{T} \int_{\Omega_{2}} \Delta w_{2} \cdot \operatorname{trl}\left(w_{2}\right) d x d t \\
\geq & -\varepsilon \int_{0}^{T} \int_{\Omega_{2}} a\left(w_{2}, w_{2}\right) d x d t-C_{\varepsilon} \int_{0}^{T} \int_{\Omega_{2}}\left(\left|l\left(w_{2}\right)\right|_{T_{x}}^{2}+\left(\operatorname{trl}\left(w_{2}\right)\right)^{2}\right) d x d t, \\
& \int_{0}^{T} \int_{\Omega_{2}}\left[2(1-\mu) D^{2} w_{2}\left(D(\operatorname{div} H), D w_{2}\right)+2 \mu \Delta w_{2}\left\langle D(\operatorname{div} H), D w_{2}\right\rangle\right] d x d t \\
\geq & -\varepsilon \int_{0}^{T} \int_{\Omega_{2}} a\left(w_{2}, w_{2}\right) d x d t-C_{\varepsilon} \int_{0}^{T}\left\|D w_{2}\right\|^{2} d t,
\end{aligned}
$$

and

$$
\int_{0}^{T} \int_{\Omega_{2}} w_{2} a\left(\operatorname{div} H, w_{2}\right) d x d t \geq-\varepsilon \int_{0}^{T} \int_{\Omega_{2}} a\left(w_{2}, w_{2}\right) d x d t-C_{\varepsilon} \int_{0}^{T}\left\|w_{2}\right\|^{2} d t,
$$

where $\varepsilon>0$ can be an arbitrary small constant and $\|\cdot\|$ denotes the $L^{2}\left(\Omega_{2}\right)$ norm. Due to 
the assumption (1.5) we have

$$
\begin{aligned}
& 4(1-\mu) \int_{0}^{T} \int_{\Omega_{2}} D H\left(D^{2} w_{2}, D^{2} w_{2}\right) d x d t+4 \mu \int_{0}^{T} \int_{\Omega_{2}} D H\left(\Delta w_{2}, \Delta w_{2}\right) d x d t \\
\geq & 4(1-\mu) \rho \int_{0}^{T} \int_{\Omega_{2}}\left|D^{2} w_{2}\right|_{T_{x}}^{2} d x d t+4 \mu \rho \int_{0}^{T} \int_{\Omega_{2}}\left(\Delta w_{2}\right)^{2} d x d t \\
\geq & 4 \rho \int_{0}^{T} \int_{\Omega_{2}} a\left(w_{2}, w_{2}\right) d x d t .
\end{aligned}
$$

Substituting inequalities (3.3)-(3.6) to equality (3.2) yields

$$
\begin{aligned}
& \int_{0}^{T} \int_{\Gamma_{2} \cup S}\left(\mathfrak{B}_{2}\left(w_{2}\right)+\mathfrak{B}_{2}^{\prime}\left(w_{2}\right)\right) d \sigma d t \\
\geq & \frac{3}{2} \rho \int_{0}^{T} \int_{\Omega_{2}}\left[\left(\partial_{t} w_{2}\right)^{2}+a\left(w_{2}, w_{2}\right)\right] d x d t-\varepsilon\left(E_{2}(T)+E_{2}(0)\right) \\
& -C_{\varepsilon}\left(\left\|D w_{2}(0)\right\|^{2}+\left\|D w_{2}(T)\right\|^{2}+\left\|w_{2}(0)\right\|^{2}+\left\|w_{2}(T)\right\|^{2}\right) \\
& -C_{\varepsilon} \int_{0}^{T}\left(\left\|w_{2}\right\|^{2}+\left\|D w_{2}\right\|^{2}\right) d t
\end{aligned}
$$

where we notice that we shall take $\varepsilon<\frac{\rho}{2}$.

Step 2. Estimates of the wave part on $\Omega_{1}$.

Similarly, using Lemma 2.6 and 2.7 we obtain

$$
\begin{aligned}
& \int_{0}^{T} \int_{\Gamma_{1} \cup S}\left(\mathfrak{B}_{1}\left(w_{1}\right)+\mathfrak{B}_{1}^{\prime}\left(w_{1}\right)\right) d \sigma d t \\
= & \left.\int_{\Omega_{1}} \partial_{t} w_{1}\left(2 H\left(w_{1}\right)+\left(\operatorname{div} H-\frac{3}{2} \rho\right) w_{1}\right) d x\right|_{0} ^{T}+\frac{3}{2} \rho \int_{0}^{T} \int_{\Omega_{1}}\left[\left(\partial_{t} w_{1}\right)^{2}-\left|D w_{1}\right|^{2}\right] d x d t \\
& +2 \int_{0}^{T} \int_{\Omega_{1}} D H\left(D w_{1}, D w_{1}\right) d x d t+\int_{0}^{T} \int_{\Omega_{1}} w_{1}\left\langle D p, D w_{1}\right\rangle d x d t \\
\geq & \frac{1}{4} \rho \int_{0}^{T} \int_{\Omega_{1}}\left[\left(\partial_{t} w_{1}\right)^{2}+\left|D w_{1}\right|^{2}\right] d x d t-\varepsilon\left(E_{1}(T)+E_{1}(0)\right)-C_{\varepsilon}\left(\left\|w_{1}(0)\right\|^{2}+\left\|w_{1}(T)\right\|^{2}\right) \\
& -2 \sup _{x \in \Omega_{1}}|H|\left(E_{1}(0)+E_{1}(T)\right)-C_{\varepsilon} \int_{0}^{T}\left\|w_{1}\right\|^{2} d t
\end{aligned}
$$

where we notice that we can take $\varepsilon<\frac{\rho}{4}$.

Step 3. We combine estimates (3.7), (3.8) and Lemma 2.3 to get

$$
\begin{aligned}
& \int_{0}^{T} \int_{\Gamma_{2} \cup S}\left(\mathfrak{B}_{2}\left(w_{2}\right)+\mathfrak{B}_{2}^{\prime}\left(w_{2}\right)\right) d \sigma d t+\int_{0}^{T} \int_{\Gamma_{1} \cup S}\left(\mathfrak{B}_{1}\left(w_{1}\right)+\mathfrak{B}_{1}^{\prime}\left(w_{1}\right)\right) d \sigma d t \\
\geq & \frac{1}{2} \rho T E(0)-2 \varepsilon E(0)-4 \sup _{x \in \Omega_{1}}|H| E(0)+\mathfrak{L}(w) \\
\geq & c_{T} E(0)+\mathfrak{L}(w),
\end{aligned}
$$


for $T>T_{0} \equiv 8 \rho^{-1} \sup _{x \in \Omega_{1}}|H|$, where we have

$$
\begin{aligned}
\mathfrak{L}(w)= & -C_{\varepsilon}\left(\left\|D w_{2}(0)\right\|^{2}+\left\|D w_{2}(T)\right\|^{2}+\left\|w_{2}(0)\right\|^{2}+\left\|w_{2}(T)\right\|^{2}\right) \\
& -C_{\varepsilon} \int_{0}^{T}\left(\left\|w_{2}\right\|^{2}+\left\|D w_{2}\right\|^{2}\right) d t-C_{\varepsilon}\left(\left\|w_{1}(0)\right\|^{2}+\left\|w_{1}(T)\right\|^{2}\right) \\
& -C_{\varepsilon} \int_{0}^{T}\left\|w_{1}\right\|^{2} d t
\end{aligned}
$$

which is a lower order term with respect to the energy $E(t)$.

Next, we deal with the left hand side of the inequality (3.9). On $S$ we have

$$
\begin{aligned}
H\left(w_{1}\right) & =\left\langle D w_{1}, H\right\rangle=\partial_{\nu_{1}} w_{1}\left\langle H, \nu_{1}\right\rangle+\partial_{\tau_{1}} w_{1}\left\langle H, \tau_{1}\right\rangle=\partial_{\tau_{1}} w_{1}\left\langle H, \tau_{1}\right\rangle \\
& =\partial_{\tau_{2}} w_{2}\left\langle H, \tau_{2}\right\rangle=\left\langle D w_{1}, H\right\rangle=H\left(w_{2}\right)
\end{aligned}
$$

where we notice that $\langle H, \nu\rangle=0$ and $w_{1}=w_{2}$ on $S$. Combining identity (3.11) and the boundary condition on the interface $S$ yields

$$
\left.\mathfrak{B}_{1}\left(w_{1}\right)\right|_{S}=\left.2 \partial_{\nu} w_{1} H\left(w_{1}\right)\right|_{S}=\left.2 B_{2}\left(w_{2}\right) H\left(w_{2}\right)\right|_{S}=-\left.\mathfrak{B}_{2}\left(w_{2}\right)\right|_{S} .
$$

Obviously we have

$$
\left.\mathfrak{B}_{1}^{\prime}\left(w_{1}\right)\right|_{S}=\left.\left(\operatorname{div} H-\frac{3}{2} \rho\right) w_{1} \partial_{\nu} w_{1}\right|_{S}=\left.\left(\operatorname{div} H-\frac{3}{2} \rho\right) w_{2} B_{2}\left(w_{2}\right)\right|_{S}=-\left.\mathfrak{B}_{2}^{\prime}\left(w_{2}\right)\right|_{S}
$$

Thus we obtain

$$
\int_{0}^{T} \int_{S}\left(\mathfrak{B}_{2}\left(w_{2}\right)+\mathfrak{B}_{2}^{\prime}\left(w_{2}\right)\right) d \sigma d t+\int_{0}^{T} \int_{S}\left(\mathfrak{B}_{1}\left(w_{1}\right)+\mathfrak{B}_{1}^{\prime}\left(w_{1}\right)\right) d \sigma d t=0 .
$$

On the other hand we have on $\Gamma_{1}$ that

$$
\begin{aligned}
H\left(w_{1}\right)=\partial_{\nu_{1}} w_{1}\left\langle H, \nu_{1}\right\rangle+\partial_{\tau_{1}} w_{1}\left\langle H, \tau_{1}\right\rangle & =\partial_{\nu_{1}} w_{1}\left\langle H, \nu_{1}\right\rangle \\
\left|D w_{1}\right|^{2} & =\left(\partial_{\nu_{1}} w_{1}\right)^{2}
\end{aligned}
$$

which yields

$$
\left.\mathfrak{B}_{1}\left(w_{1}\right)\right|_{\Gamma_{1}}=\left(\partial_{\nu_{1}} w_{1}\right)^{2}\left\langle H, \nu_{1}\right\rangle .
$$

Due to the boundary condition $w_{2}=\partial_{\nu_{2}} w_{2}=0$ on $\Gamma_{2}$ we have

$$
D w_{2}=\left(\partial_{\nu_{2}} w_{2}\right) \nu_{2}+\left(\partial_{\tau_{2}} w_{2}\right) \tau_{2}=0, \quad H\left(w_{2}\right)=0
$$

which yield on $\Gamma_{2}$

$$
D^{2} w_{2}\left(\tau_{2}, \nu_{2}\right)=\left\langle D_{\tau_{2}}\left(D w_{2}\right), \nu_{2}\right\rangle=\left\langle 0, \nu_{2}\right\rangle=0
$$

and

$$
D^{2} w_{2}\left(\tau_{2}, \tau_{2}\right)=\left\langle D_{\tau_{2}}\left(D w_{2}\right), \tau_{2}\right\rangle=\left\langle 0, \tau_{2}\right\rangle=0
$$


Therefore on $\Gamma_{2}$ we have

$$
\begin{aligned}
\mathfrak{B}_{2}\left(w_{2}\right) & =2 B_{1}\left(w_{2}\right) \partial_{\nu_{2}}\left(H\left(w_{2}\right)\right)-a\left(w_{2}, w_{2}\right)\left\langle H, \nu_{2}\right\rangle \\
& =\left(D^{2} w_{2}\left(\nu_{2}, \nu_{2}\right)\right)^{2}\left\langle H, \nu_{2}\right\rangle
\end{aligned}
$$

where we notice on $\Gamma_{2}$ that

$$
\begin{aligned}
\partial_{\nu_{2}}\left(H\left(w_{2}\right)\right) & =D^{2} w_{2}\left(H, \nu_{2}\right)+D w_{2}\left(D_{\nu_{2}} H\right) \\
& =D^{2} w_{2}\left(\left(\left\langle H, \nu_{2}\right\rangle \nu_{2}+\left\langle H, \tau_{2}\right\rangle \tau_{2}\right), \nu_{2}\right)=\left\langle H, \nu_{2}\right\rangle D^{2} w_{2}\left(\nu_{2}, \nu_{2}\right)
\end{aligned}
$$

It's easy to know

$$
\left.\mathfrak{B}_{1}^{\prime}\left(w_{1}\right)\right|_{\Gamma_{1}}=\left.\mathfrak{B}_{2}^{\prime}\left(w_{2}\right)\right|_{\Gamma_{2}}=0
$$

Finally we get

$$
\begin{aligned}
& \int_{0}^{T} \int_{\Gamma_{1}}\left(\mathfrak{B}_{2}\left(w_{2}\right)+\mathfrak{B}_{2}^{\prime}\left(w_{2}\right)\right) d \sigma d t+\int_{0}^{T} \int_{\Gamma_{2}}\left(\mathfrak{B}_{1}\left(w_{1}\right)+\mathfrak{B}_{1}^{\prime}\left(w_{1}\right)\right) d \sigma d t \\
= & \int_{0}^{T} \int_{\Gamma_{1}}\left(\partial_{\nu_{1}} w_{1}\right)^{2}\left\langle H, \nu_{1}\right\rangle d \sigma d t+\int_{0}^{T} \int_{\Gamma_{2}}\left(D^{2} w_{2}\left(\nu_{2}, \nu_{2}\right)\right)^{2}\left\langle H, \nu_{2}\right\rangle d \sigma d t .
\end{aligned}
$$

Combining (3.9), (3.14) and (3.22) to obtain

$$
\begin{aligned}
& \int_{0}^{T} \int_{\Gamma_{1}}\left(\partial_{\nu_{1}} w_{1}\right)^{2}\left\langle H, \nu_{1}\right\rangle d \sigma d t+\int_{0}^{T} \int_{\Gamma_{2}}\left(D^{2} w_{2}\left(\nu_{2}, \nu_{2}\right)\right)^{2}\left\langle H, \nu_{2}\right\rangle d \sigma d t \\
\geq & c_{T} E(0)+\mathfrak{L}(w) .
\end{aligned}
$$

Finally, the lower order term $\mathfrak{L}(w)$ can be absorbed using a compactness-uniqueness argument in the literature (see [13] and many others). Consequently the inequality (3.1) follows considering the definition of $\Gamma_{0}^{i}$ in (1.6).

Acknowledgements The authors wish to thank the editor and the referee for their useful comments.

\section{References}

[1] K. Ammari and S. Nicaise, Stabilization of a transmission wave/plate equation, Journal of Differential Equations, 249(3), 2010, 707-727.

[2] F. Hassine, Energy decay for a transmission coupled Euler-Bernoulli and wave equation with force and moment feedback, arXiv:1303.2831v1[math.AP], 2013.

[3] S. Chai, and K. Liu, Observability inequalities for the transmission of shallow shells. Systems Control Lett. 55 (2006), no. 9, 726C735.

[4] S. J. Feng, and D. X. Feng, Nonlinear internal damping of wave equations with variable coefficients, Acta Math. Sin., Engl. Ser., 20(6), 2004, 1057-1072. 
[5] R. E. Green and H. Wu, $\mathrm{C}^{\infty}$ convex functions and manifolds of positive curvature, Acta Math., 137(1), 1976, 209-245.

[6] R. Gulliver, I. Lasiecka, W. Littman, et al, The case for differential geometry in the control of single and coupled PDEs: the structural acoustic chamber, Geometric Methods in Inverse Problems and PDE Control, 73-181, IMA Vol. Math. Appl., 137, SpringerVerlag, New York, 2004.

[7] B. Z. Guo, and Z. C. Shao, On well-posedness, regularity and exact controllability for problems of transmission of plate equation with variable coefficients, Quarterly of Applied Mathematics, Vol LXV, no.4, 2007, 705-736.

[8] Y. Guo, and P. F. Yao, Stabilization of Euler Bernoulli plate equation with variable coefficients by nonlinear boundary feedback, J. Math. Anal. Appl., 317, 2006, 50-70.

[9] B. V. Kapitonov, Uniform stabilization and simultaneous exact boundary controllability for a pair of hyperbolic systems, Siberian Math. J., 35 (1994), 722-734.

[10] J. Lagnese, Boundary controllability in problems of transmission for a class of second order hyperbolic systems, ESAIM Control Optim. Calc., 1997, 2: 343-357.

[11] I. Lasiecka, J. L. Lions, and R. Triggiani, Non homogeneous boundary value problems for second-order hyperbolic operators, J. Math. Pures Appl., 65(1986), 149-192.

[12] I. Lasiecka and R. Triggiani, Exact controllability of the wave equation with Neumann boundary control, Appl. Math. and Optimiz., 19 (1989), 243-290.

[13] I. Lasiecka and R. Triggiani, Global exact controllability of semilinear wave equations by a double compactness/uniqueness argument, Discrete and Continuous Dynamical Systems, Vol. Supplement, pp. 556-566 (2005).

[14] I. Lasiecka, R. Triggiani, and P. F. Yao, Inverse/observability estimates for second-order hyperbolic equations with variable coefficients, J. Math. Anal. Appl., 235(1), 1999, 1357.

[15] J. L. Lions, Contrôlabilité Exacte Perturbations et Stabilization de Systèmes Distribués, Tome 1, Contrôlabilité Exacte Masson, Paris, 1988.

[16] W. Liu, G. H. Williams, Exact controllability for problems of transmission of the plate equation with lower-order terms, Quart. Appl. Math., 58 (2000), no. 1, 37-68.

[17] W. Liu, Stabilization and controllability for the transmission wave equation, IEEE Transactions on Automation Control, 2001, 46(12), 1900-1907.

[18] S. Nicaise, Boundary exact controllability of interface problems with singularities, II: addition of internal control, SIAM, J., Control Optim., 35 (1997), 585C603. 
[19] H. P. Oquendo, Nonlinear boundary stabilization for a transmission problem in elasticity, Nonlinear Analysis, 2003, 52(4), 1331-1354.

[20] H. Wu, C.L. Shen, Y.L. Yu, An Introduction to Riemannian Geometry, Univ. of Beijing, 1989 (in Chinese).

[21] P. F. Yao, On the observatility inequality for exact controllability of wave equations with variable coefficients, SIAM J. Contr. and Optim. , Vol. 37, No.5 (1999), 1568-1599.

[22] P. F. Yao, Modeling and Control in Vibrational and Structural Dynamics. A Differential Geometric Approach. Chapman and Hall/CRC Applied Mathematics and Nonlinear Science Series. CRC Press, Boca Raton, FL, 2011. xiv+405 pp. ISBN: 978-1-4398-34558.

[23] P.F.Yao, Observability inequalities for shallow shells, SIAM J. Contr. and Optim., Vol. 38, No. 6 (2000), 1729-1756.

[24] W. Zhang, Z. F. Zhang, Stabilization of transmission coupled wave and Euler-Bernoulli equations on Riemannian manifolds by nonlinear feedbacks, J. Math. Anal. Appl., 422 (2015), no. 2, 1504-1526. 